\title{
Optimization of Conversion Treatment on Austenitic Stainless Steel Using Experimental Designs
}

\author{
S. El Hajjaji, ${ }^{1}$ C. Cros, ${ }^{2}$ and L. Aries ${ }^{2}$ \\ ${ }^{1}$ Laboratoire de Spectroscopie, Modélisation Moléculaire, Matériaux et Environnement (LS3ME), Faculté des Sciences, \\ Université Med V-Agdal, Avenu Ibn Battouta, BP 1014, Rabat, Morocco \\ ${ }^{2}$ CIRIMAT-LCMIE, Université Paul Sabatier, 118 route de Narbonne, 31064 Toulouse Cedex 4, France
}

Correspondence should be addressed to S. El Hajjaji; selhajjaji@hotmail.com

Received 27 August 2013; Accepted 17 November 2013

Academic Editor: Chi Tat Kwok

Copyright (C) 2013 S. El Hajjaji et al. This is an open access article distributed under the Creative Commons Attribution License, which permits unrestricted use, distribution, and reproduction in any medium, provided the original work is properly cited.

\begin{abstract}
Conversion coating is commonly used as treatment to improve the adherence of ceramics films. The conversion coating properties depend on the structure of alloy as well as on the treatment parameters. These conversion coatings must be characterized by strong interfacial adhesion, high roughness, and high real surface area, which were measured by an electrochemical method. The influence of all the elaboration factors (temperature, time, and bath composition: sulphuric acid, thiosulphate as accelerator, propargyl alcohol as inhibitor, and surface state) and also the interactions between these factors were evaluated, using statistical experimental design. The specific surface area and optical factor $(\alpha)$ correspond to the quantitative responses. The evaluation showed, by using a designed experimental procedure, that the most important factor was "surface state." Sanded surface allows the formation of conversion coating with high real surface area. A further aim was to optimise two parameters: treatment time and temperature using Doehlert shell design and simplex method. The growth of the conversion coating is also influenced by treatment time and temperature. With such optimized conditions, the real surface area of conversion coating obtained was about $235 \mathrm{~m}^{2} / \mathrm{m}^{2}$.
\end{abstract}

\section{Introduction}

Coatings have been developed from various materials using several deposition methods [1-3]. Electrochemical deposition is an interesting technique to obtain corrosion protection coatings, but the problem for such coatings is adhesion. In previous papers [4-6], we described an original method to strengthen the interface between ceramic layer and stainless steel or super alloy substrate. This method involves three steps. In the first, the metal surface is modified by a conversion treatment in an acid bath with $\mathrm{S}^{2-}$ and acetylenic alcohol as additions, allowing the control of the conversion coating growth $[6,7]$. This pretreatment of the surface leads to a conversion coating which is very adherent, with a particular morphology, with micropores that allow deposition during the second step and contribute to the "anchoring" of the ceramic layer. In the second step, a refractory character is conferred to the surface by a cathodic treatment in a suitable bath, which induces the deposition of oxides or hydroxides with varying degrees of hydration. In the third step, a thermal treatment leads to ceramic oxides and stabilized the coating.

So, to strengthen the interface between ceramic and substrate, a specific pretreatment of the metal surface is proposed so as to form a conversion coating. The morphology of the surface is important and must present a very porous structure and a high specific area to facilitate the anchoring of the ceramic layer [5-7]. Many authors have studied the influence of different parameters in conversion treatment for different metal substrates and different applications $[5,8-$ 10].

This study was undertaken to elucidate the role of the different parameters and to optimise conversion coating on austenitic stainless steel. The parameters of conversion treatment have been studied using statistical experimental designs. The treatment process and the statistical designs are briefly reviewed before the experimental results are presented. 
TABLE 1: Chemical composition of austenitic stainless steel (wt\%).

\begin{tabular}{lccccccc}
\hline $\mathrm{C}$ & $\mathrm{Si}$ & $\mathrm{Cu}$ & $\mathrm{Mo}$ & $\mathrm{S}$ & $\mathrm{Cr}$ & $\mathrm{Ni}$ & $\mathrm{Fe}$ \\
\hline 0.031 & 0.77 & 0.06 & 0.10 & 0.007 & 18.2 & 10.3 & 70.5 \\
\hline
\end{tabular}

\section{Materials and Methods}

2.1. Conversion Treatment. Conversion coatings were prepared on an austenitic stainless steel, its composition is given in Table 1. Samples were cleaned with tetrahydrofurane (ACROS ORGANIC, purity Z99\%), washed with distilled water, and then dried in air at room temperature. Austenitic stainless steel conversion coating was obtained by chemical treatment in acid bath containing suitable additives and particularly substances containing chalcogenides such as sulphur (sulphides, thiosulphates) [4-10]. Corrosion inhibitors like acetylenic alcohols are also required to facilitate the control of film growth in order to obtain coats with specific properties [4-10].

In order to homogenize the surface hardness, samples have undergone to a surface treatment of sanding or of microball tests.

After treatment, the samples were rinsed in demineralised water, and then dried at $70^{\circ} \mathrm{C}$ for 10 minutes.

2.2. Electrochemical Study. The electrochemical measurement was performed using a Tacussel model PRT 20-02 potentiostat. A saturated calomel electrode (SCE) was used as the reference electrode and a platinum electrode was used as the counter electrode.

2.3. Methodology of Experimental Research. The objective of the methodology of experimental research (MER) is to search for an optimal strategy which allows obtaining the largest number of good quality information concerning a studied phenomenon, while carrying a limited number of experiments. These are informationally optimal mathematical schemes in which all important factors are changed simultaneously, thereby facilitating the identification of process relations as well as the location of the real process optimum.

2.3.1. Screening (Design I). The main purpose of a screening study is to identify the most influential factors and those that may be regarded as inert. Fractional factorial designs [11] were chosen to evaluate the factors that significantly influence conversion coating morphology. For each problem formulated, the first problem is the choice of the factors which are the parameters that we can control. We must choose the variation limit of these factors which determines the experimental domain. These variations may have very different orders of magnitude, so that, to be able to compare the factor effects, it is necessary to work with the code levels of variation of each factor. For the present work, we have to evaluate the influence of fix factors, each at two levels (high $(+1)$ and low $(-1)$ ). The selected parameters are listed in Table 2.

A factor is an assigned variable and the levels of the factor are the values assigned to the factor. Each experiment represents a particular point of the experimental domain and provides a measurement with one or several responses of the phenomenon in this point.

In first step we used a $2^{6-1}$ experiment; six factors each at two levels $(+1,-1)$ were investigated; 32 trials were necessary for this fractional factorial design (Table 3). A factor is an assigned variable and the levels of the factor are the values assigned to the factor. The fractional factorial design consists in expressing the estimated effects in contrast. All experiments were performed in random order and the calculation was obtained by the NEMROD program [12].

2.3.2. Optimisation. In the second step, once the most significant factors have been identified, the next step is to optimise the process with respect to these factors. In this work, we used a "Doehlert uniform shell design" [13-15] and a simplex method [15].

In the present work, we studied two factors: treatment time $\left(X_{1}\right)$ and temperature $\left(X_{2}\right)$, requiring that six coefficients be determined as follows:

$$
y=b_{0}+b_{1} X_{1}+b_{2} X_{2}+b_{11} X_{1}^{2}+b_{22} X_{2}^{2}+b_{12} X_{1} X_{2} .
$$

The experimental design is presented in Table 4. Their variation domains were determined in preliminary experiments (Table 4). To minimize the effect of uncontrolled factors and time variations, all experiments were performed in random order.

2.4. Measurement of Responses. In order to show the effect of each factor, the studied responses are the real surface area or specific surface area (SS) of the conversion coating expressed in $\mathrm{m}^{2} / \mathrm{m}^{2}$ and its optical properties $(\alpha)$.

2.4.1. Measurement of Specific Area of the Coating. The main characteristic of conversion coatings is their high porosity. The porous character was evaluated using cyclic voltammetry to obtain the real surface area. This method involves application of a potential $E$, which varies with time to an electrode, between -0.2 and $-1.5 \mathrm{~V} / \mathrm{SCE}$ in a $1 \mathrm{M}$ sodium sulphate medium. The scanning rate was $20 \mathrm{mV} / \mathrm{s}$. The measurements were performed with the three-electrode technique. This measurement assumes the formation of a monolayer of adsorbed hydrogen and one atom of hydrogen is taken as occupying $10 \AA^{2}[16,17]$. The surface area for $1 \mathrm{~cm}^{2}$ was given below:

$$
\mathrm{SS}=\frac{\mathrm{QN} 10 \AA^{2}}{n F}
$$

where $Q$ is the quantity of electricity (coulomb) corresponding to the anodic peak area. $N$ is Avogadro's number. $N$ is the number of electron $\left(\mathrm{H}^{+}+1 \mathrm{e}^{-} \rightarrow \mathrm{H}_{\mathrm{ads}}, n=1\right)$ and $F=96500 \mathrm{C} \mathrm{mol}^{-1}$.

2.4.2. Measurement of Optical Property $(\alpha)$. The total hemispheric solar adsorption factor $\alpha$ (ration of the energy adsorbed by the surface to the incident solar energy) was measured with an EL510 alpha meter (Elan Informatique). 
TABLE 2: Factors and their levels for the experiments.

\begin{tabular}{|c|c|c|c|c|c|c|c|c|c|c|c|}
\hline \multicolumn{2}{|c|}{$\begin{array}{c}\mathrm{H}_{2} \mathrm{SO}_{4} \% \\
\left(X_{1}\right)\end{array}$} & \multicolumn{2}{|c|}{$\begin{array}{c}T\left({ }^{\circ} \mathrm{C}\right) \\
\left(X_{2}\right)\end{array}$} & \multicolumn{2}{|c|}{$\begin{array}{c}\mathrm{Na}_{2} \mathrm{~S}_{2} \mathrm{O}_{3}, 5 \mathrm{H}_{2} \mathrm{O} \\
\mathrm{g} / \mathrm{L}\left(X_{3}\right)\end{array}$} & \multicolumn{2}{|c|}{$\begin{array}{c}\mathrm{C}_{3} \mathrm{H}_{4} \mathrm{O} \% \\
\left(X_{4}\right)\end{array}$} & \multicolumn{2}{|c|}{$\begin{array}{l}\text { Treatment time (min) } \\
\qquad\left(X_{5}\right)\end{array}$} & \multicolumn{2}{|c|}{$\begin{array}{l}\text { Surface state } \\
\qquad\left(X_{6}\right)\end{array}$} \\
\hline-1 & +1 & -1 & +1 & -1 & +1 & -1 & +1 & -1 & +1 & -1 & +1 \\
\hline 0.2 & 2 & 40 & 70 & 0.5 & 1.5 & 0 & 0.25 & 10 & 30 & Sanded & Microball \\
\hline
\end{tabular}

TABLE 3: Fractional factorial design $2^{6-1}$ : theoretical values of coded variables.

\begin{tabular}{|c|c|c|c|c|c|c|}
\hline No. & $X_{1}$ & $X_{2}$ & $X_{3}$ & $X_{4}$ & $X_{5}$ & $X_{6}$ \\
\hline 1 & -1 & -1 & -1 & -1 & -1 & 1 \\
\hline 2 & 1 & -1 & -1 & -1 & -1 & -1 \\
\hline 3 & -1 & 1 & -1 & -1 & -1 & -1 \\
\hline 4 & 1 & 1 & -1 & -1 & -1 & 1 \\
\hline 5 & -1 & -1 & 1 & -1 & -1 & -1 \\
\hline 6 & 1 & -1 & 1 & -1 & -1 & 1 \\
\hline 7 & -1 & 1 & 1 & -1 & -1 & 1 \\
\hline 8 & 1 & 1 & 1 & -1 & -1 & -1 \\
\hline 9 & -1 & -1 & -1 & 1 & -1 & 1 \\
\hline 10 & 1 & -1 & -1 & 1 & -1 & -1 \\
\hline 11 & -1 & 1 & -1 & 1 & -1 & -1 \\
\hline 12 & 1 & 1 & -1 & 1 & -1 & 1 \\
\hline 13 & -1 & -1 & 1 & 1 & -1 & -1 \\
\hline 14 & 1 & -1 & 1 & 1 & -1 & 1 \\
\hline 15 & -1 & 1 & 1 & 1 & -1 & 1 \\
\hline 16 & 1 & 1 & 1 & 1 & -1 & -1 \\
\hline 17 & -1 & -1 & -1 & -1 & 1 & -1 \\
\hline 18 & 1 & -1 & -1 & -1 & 1 & 1 \\
\hline 19 & -1 & 1 & -1 & -1 & 1 & 1 \\
\hline 20 & 1 & 1 & -1 & -1 & 1 & -1 \\
\hline 21 & -1 & -1 & 1 & -1 & 1 & 1 \\
\hline 22 & 1 & -1 & 1 & -1 & 1 & -1 \\
\hline 23 & -1 & 1 & 1 & -1 & 1 & -1 \\
\hline 24 & 1 & 1 & 1 & -1 & 1 & 1 \\
\hline 25 & -1 & -1 & -1 & 1 & 1 & -1 \\
\hline 26 & 1 & -1 & -1 & 1 & 1 & 1 \\
\hline 27 & -1 & 1 & -1 & 1 & 1 & 1 \\
\hline 28 & 1 & 1 & -1 & 1 & 1 & -1 \\
\hline 29 & -1 & -1 & 1 & 1 & 1 & 1 \\
\hline 30 & 1 & -1 & 1 & 1 & 1 & -1 \\
\hline 31 & -1 & 1 & 1 & 1 & 1 & -1 \\
\hline 32 & 1 & 1 & 1 & 1 & 1 & 1 \\
\hline
\end{tabular}

2.5. Measurement of Electrochemical Impedance. EIS measurements were performed using EGG PAR apparatus model 16310. Impedance spectra were obtained in the frequency range of $10 \mathrm{KHz}$ to $10 \mathrm{MHz}$. AC amplitude was $5 \mathrm{mV}$. Experiments were performed in aqueous aerated solution of $1 \mathrm{M}$ $\mathrm{Na}_{2} \mathrm{SO}_{4}$ at $25^{\circ} \mathrm{C}$.
TABLE 4: Doehlert design: theoretical values of coded variables and their levels for the experiments.

\begin{tabular}{lcccc}
\hline No. & $X_{1}$ & $X_{2}$ & Treatment time $(\mathrm{s})$ & $T\left({ }^{\circ} \mathrm{C}\right)$ \\
\hline 1 & 1 & 0 & 300 & 55.00 \\
2 & -1 & 0 & 180 & 55.00 \\
3 & 0.5 & 0.866 & 270 & 60.02 \\
4 & -0.5 & -0.866 & 210 & 49.98 \\
5 & 0.5 & -0.866 & 270 & 49.98 \\
6 & -0.5 & 0.866 & 210 & 60.02 \\
7 & 0 & 0 & 240 & 55.00 \\
\hline
\end{tabular}

\section{Results and Discussion}

3.1. Determination of the Significant Factors (FFDs $2^{6-1}$ ). Experimental treatment conditions and the specific surface area and $\alpha$ of the as-prepared conversion coatings are shown in Table 5. Values of the 11 contrasts were computed with $\mathrm{NEMROD}^{\mathrm{w}}$ software and are given in Table 6. The study of these results indicates that the process can be explained by the strong effects corresponding to sulphuric acid, alcohol concentrations, and to the effect of the interaction of alcohol concentration with acid concentration and temperature. Figure 1 serves as an illustration for studying these interactions.

The effect of alcohol depends on the level of temperature. The results show that the increase of propargyl alcohol concentration for a low temperature has no effect on the real surface. But, at high temperature, the best result corresponds to a low alcohol concentration ( $\mathrm{SS}=115 \mathrm{~m}^{2} / \mathrm{m}^{2}$ ).

The effect of alcohol depends on the level of acid. At low or high alcohol concentration, the responses are highly influenced by the variation of acid concentration and the best result corresponds to a low concentrations of sulfuric acid and propargyl alcohol $\left(\mathrm{SS}=129 \mathrm{~m}^{2} / \mathrm{m}^{2}\right)$. One other factor also has a significant main effect and does not display any interaction: surface state $\left(b_{6}=-30.4\right)$. We can therefore state that the value of SS is higher if the surface is at level -1 . In this case, sand surface is the surface that serves to obtain the highest SS.

A great real surface area of the conversion coating is achieved under the following experimental conditions.

Sulphuric acid concentration $0.2 \%$.

Thiosulphate concentration: 0.5 or $1.5 \mathrm{~g} / \mathrm{L}$.

Alcohol concentration: $0 \%$.

Temperature: $70^{\circ} \mathrm{C}$.

Treatment time: $10 \mathrm{~min}$ or $30 \mathrm{~min}$. 
TABLE 5: Fractional factorial design $\left(2^{6-1}\right)$ : variables, their levels, and data of the responses (SS and $\alpha$ ).

\begin{tabular}{|c|c|c|c|c|c|c|c|c|}
\hline No. & $\begin{array}{l}\mathrm{H}_{2} \mathrm{SO}_{4} \% \\
\left(X_{1}\right)\end{array}$ & $\begin{array}{c}T\left({ }^{\circ} \mathrm{C}\right) \\
\left(X_{2}\right)\end{array}$ & $\begin{array}{l}\mathrm{Na}_{2} \mathrm{~S}_{2} \mathrm{O}_{8} \\
\mathrm{~g} / \mathrm{L}\left(X_{3}\right)\end{array}$ & $\begin{array}{c}\mathrm{C}_{3} \mathrm{H}_{4} \mathrm{O} \% \\
\left(X_{4}\right)\end{array}$ & $\begin{array}{l}\text { Treatment time (min) } \\
\qquad\left(X_{5}\right)\end{array}$ & $\begin{array}{l}\text { Surface state } \\
\qquad\left(X_{6}\right)\end{array}$ & $\begin{array}{c}\text { SS } \\
\left(\mathrm{m}^{2} / \mathrm{m}^{2}\right)\end{array}$ & $\alpha$ \\
\hline 1 & 0.2 & 40 & 0.5 & 0 & 10 & Microball & 40.20 & 0.76 \\
\hline 2 & 2 & 40 & 0.5 & 0 & 10 & Sanded & 15.10 & 0.45 \\
\hline 3 & 0.2 & 70 & 0.5 & 0 & 10 & Sanded & 226.00 & 0.94 \\
\hline 4 & 2 & 70 & 0.5 & 0 & 10 & Microball & 8.00 & 0.83 \\
\hline 5 & 0.2 & 40 & 1.5 & 0 & 10 & Sanded & 131.20 & 0.90 \\
\hline 6 & 2 & 40 & 1.5 & 0 & 10 & Microball & 6.40 & 0.27 \\
\hline 7 & 0.2 & 70 & 1.5 & 0 & 10 & Microball & 160.10 & 0.92 \\
\hline 8 & 2 & 70 & 1.5 & 0 & 10 & Sanded & 97.00 & 0.89 \\
\hline 9 & 0.2 & 40 & 0.5 & 0.25 & 10 & Microball & 19.70 & 0.54 \\
\hline 10 & 2 & 40 & 0.5 & 0.25 & 10 & Sanded & 83.70 & 0.31 \\
\hline 11 & 0.2 & 70 & 0.5 & 0.25 & 10 & Sanded & 65.70 & 0.75 \\
\hline 12 & 2 & 70 & 0.5 & 0.25 & 10 & Microball & 44.90 & 0.66 \\
\hline 13 & 0.2 & 40 & 1.5 & 0.25 & 10 & Sanded & 95.30 & 0.71 \\
\hline 14 & 2 & 40 & 1.5 & 0.25 & 10 & Microball & 44.70 & 0.46 \\
\hline 15 & 0.2 & 70 & 1.5 & 0.25 & 10 & Microball & 29.20 & 0.76 \\
\hline 16 & 2 & 70 & 1.5 & 0.25 & 10 & Sanded & 102.40 & 0.61 \\
\hline 17 & 0.2 & 40 & 0.5 & 0 & 30 & Sanded & 164.70 & 0.91 \\
\hline 18 & 2 & 40 & 0.5 & 0 & 30 & Microball & 5.30 & 0.75 \\
\hline 19 & 0.2 & 70 & 0.5 & 0 & 30 & Microball & 142.80 & 0.94 \\
\hline 20 & 2 & 70 & 0.5 & 0 & 30 & Sanded & 136.10 & 0.96 \\
\hline 21 & 0.2 & 40 & 1.5 & 0 & 30 & Microball & 46.70 & 0.94 \\
\hline 22 & 2 & 40 & 1.5 & 0 & 30 & Sanded & 139.60 & 0.94 \\
\hline 23 & 0.2 & 70 & 1.5 & 0 & 30 & Sanded & 119.50 & 0.91 \\
\hline 24 & 2 & 70 & 1.5 & 0 & 30 & Microball & 37.10 & 0.92 \\
\hline 25 & 0.2 & 40 & 0.5 & 0.25 & 30 & Sanded & 47.40 & 0.77 \\
\hline 26 & 2 & 40 & 0.5 & 0.25 & 30 & Microball & 54.20 & 0.73 \\
\hline 27 & 0.2 & 70 & 0.5 & 0.25 & 30 & Microball & 10.20 & 0.49 \\
\hline 28 & 2 & 70 & 0.5 & 0.25 & 30 & Sanded & 107.80 & 0.82 \\
\hline 29 & 0.2 & 40 & 1.5 & 0.25 & 30 & Microball & 60.50 & 0.79 \\
\hline 30 & 2 & 40 & 1.5 & 0.25 & 30 & Sanded & 146.40 & 0.84 \\
\hline 31 & 0.2 & 70 & 1.5 & 0.25 & 30 & Sanded & 25.60 & 0.70 \\
\hline 32 & 2 & 70 & 1.5 & 0.25 & 30 & Microball & 22.40 & 0.88 \\
\hline
\end{tabular}

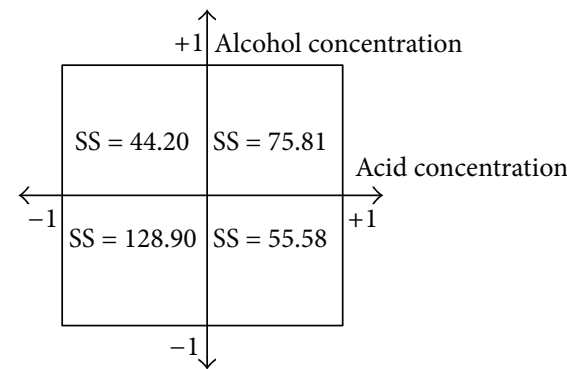

(a)

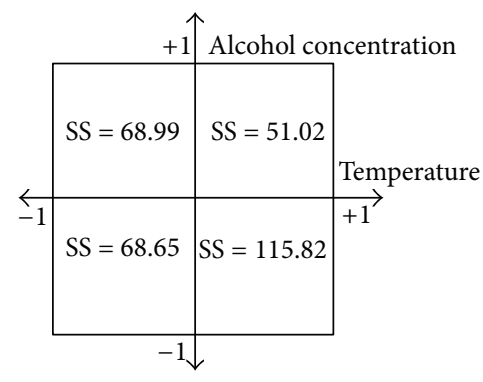

(b)

Figure 1: Illustration of interactions $b_{14}$ and $b_{24}$ between (a) sulphuric acid concentration $\left(X_{1}\right)$-alcohol concentration $\left(X_{4}\right)$ and (b) temperature $\left(X_{2}\right)$-alcohol concentration $\left(X_{4}\right)$. 


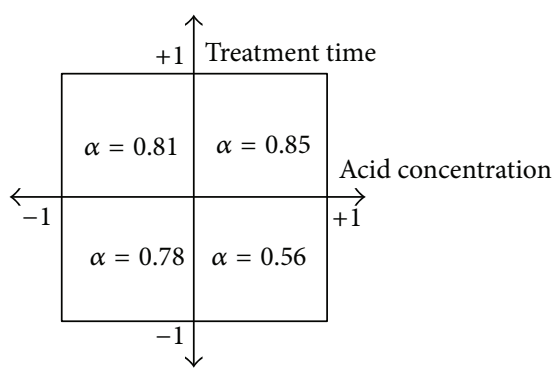

(a)

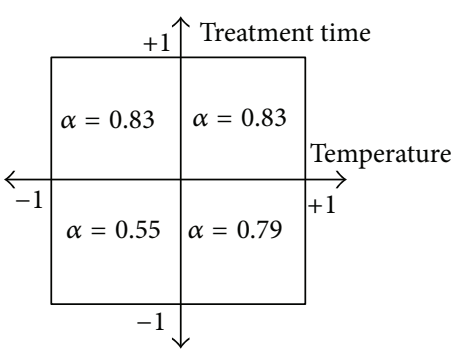

(b)

FIGURE 2: Illustration of interactions $b_{15}$ and $b_{25}$ between (a) sulphuric acid concentration $\left(X_{1}\right)$ - treatment time $\left(X_{5}\right)$ and (b) temperature $\left(X_{2}\right)$-treatment time $\left(X_{4}\right)$.

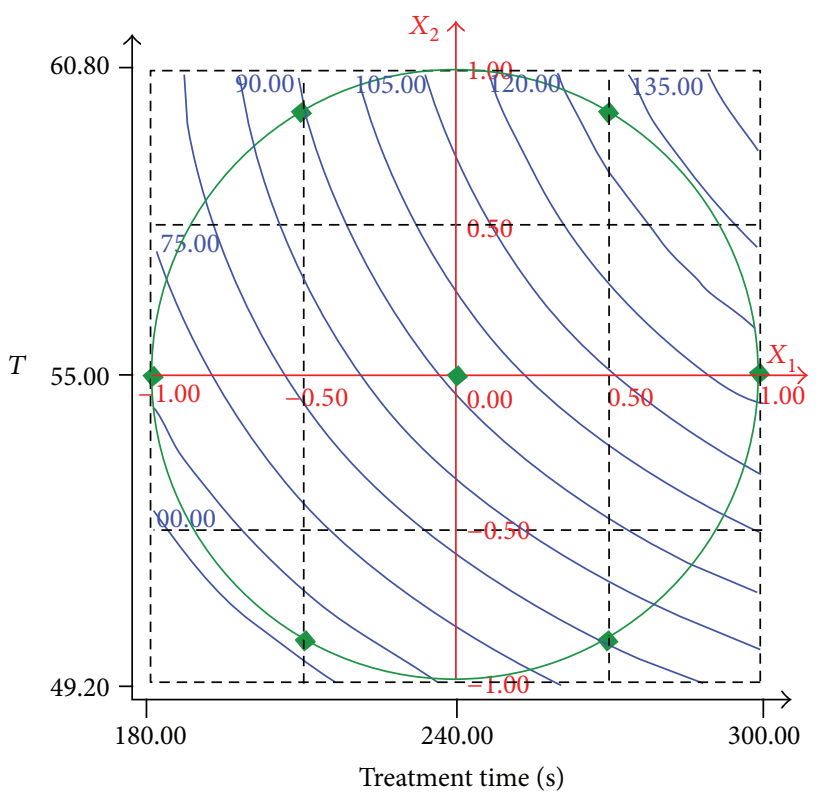

(a)

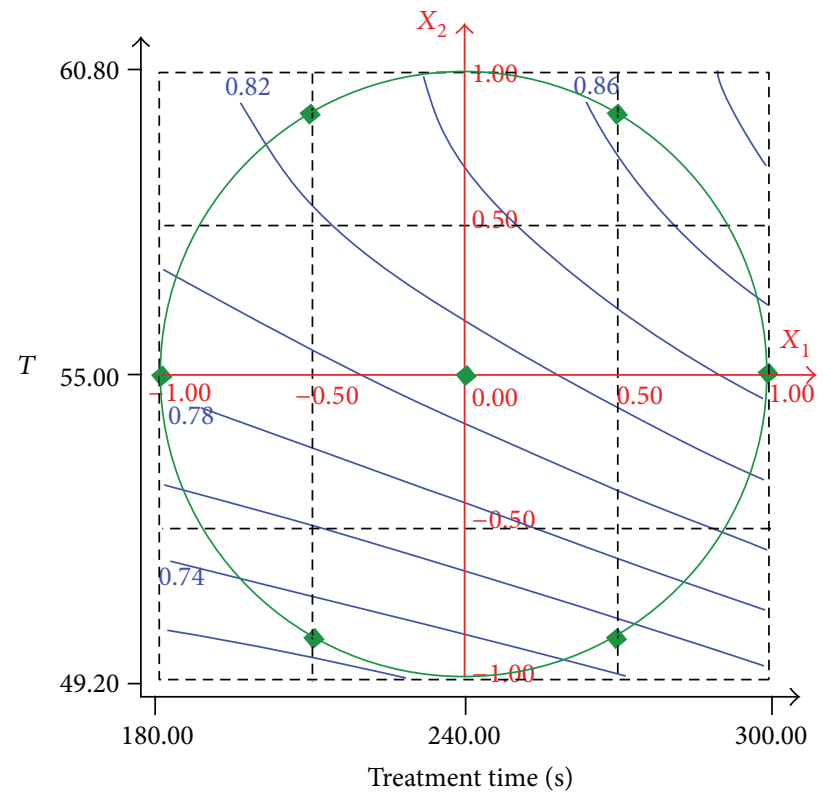

(b)

Figure 3: Response surface of the real surface area (a) and $\alpha$ (b) versus $X_{1}$ (treatment time) and $X_{2}$ (temperature).

For $\alpha$ response, the significant one here is also alcohol concentration that must be at level $(-1)$. Two interactions $\left(b_{15}\right.$ and $\left.b_{25}\right)$, corresponding to the interaction acid-treatment time and temperature-treatment time, respectively, have a significant effect and can be illustrated as in Figure 2. It emerges from these two interactions that the acid concentration, treatment time, and temperature must be at level $(-1)$.

We therefore decided to work in the following conditions:

Acid concentration: $0.2 \%(-1)$.

Thiosulphate concentration: $0.5 \mathrm{~g} / \mathrm{L}(-1)$.

Alcohol concentration: $0(-1)$.

Surface sanded surface: $(-1)$.

And in these conditions, we focused essentially on the effect of the treatment time and temperature using a Doehlert uniform shell design for two parameters, and in which a second order response surface is fitted to the experimental result by least squares multiple regressions by NEMROD software.

3.2. Doehlert Shell Design and Simplex Matrix. The results obtained according to Doehlert's matrix are given in Table 7 . The experimental domain was determined from their best levels.

Processing of the data led to the estimation of six coefficients for the polynomial equation for each response as follows:

$$
\begin{aligned}
\text { SS }=99+ & 27.2 X_{1}+24 X_{2}-3 X_{1}^{2}-2.7 X_{2}^{2}+7.5 X_{1} X_{2}, \\
\alpha= & 0.81+0.03 X_{1}+0.06 X_{2}+0.005 X_{1}^{2} \\
& -0.03 X_{2}^{2}+0.006 X_{1} X_{2}
\end{aligned}
$$

( $X_{1}$ : treatment time, $X_{2}$ : temperature). 
TABLE 6: Main and interactions effects calculated from factorial fractional design $2^{6-1}$.

\begin{tabular}{lcc}
\hline$b_{i}$ & Estimates (for responses SS) & Estimates (for responses $\alpha$ ) \\
\hline$b_{0}$ & 76.1 & 0.75 \\
$b_{1}$ & -10.4 & -0.04 \\
$b_{2}$ & 7.3 & 0.06 \\
$b_{3}$ & 2.9 & 0.03 \\
$b_{4}$ & $-\mathbf{1 6 . 1}$ & $-\mathbf{0 . 0 8}$ \\
$b_{5}$ & 3.0 & $\mathbf{0 . 0 8}$ \\
$b_{6}$ & $-\mathbf{3 0 . 4}$ & 0.02 \\
$b_{14}$ & $\mathbf{2 6 . 2}$ & 0.03 \\
$b_{24}$ & $-\mathbf{1 6 . 3}$ & -0.03 \\
$b_{15}$ & 12.4 & $\mathbf{0 . 0 7}$ \\
$b_{25}$ & -11.3 & -0.06 \\
\hline
\end{tabular}

The bold font refers to the most important values according to their levels as it is explain in the text.

TABLE 7: Doehlert design: results for each experiment.

\begin{tabular}{lcccc}
\hline No. & Time $(\mathrm{s})$ & $\mathrm{T}\left({ }^{\circ} \mathrm{C}\right)$ & $\mathrm{SS}\left(\mathrm{m}^{2} / \mathrm{m}^{2}\right)$ & $\alpha$ \\
\hline 1 & 300 & 55.00 & 120 & 0.85 \\
2 & 180 & 55.00 & 72 & 0.78 \\
3 & 270 & 60.02 & 134 & 0.86 \\
4 & 210 & 49.98 & 59 & 0.73 \\
5 & 270 & 49.98 & 86 & 0.75 \\
6 & 210 & 60.02 & 94 & 0.83 \\
7 & 240 & 55.00 & 99 & 0.81 \\
\hline
\end{tabular}

Figure 3 represents the variation of responses SS (Figure 3(a)) and $\alpha$ (Figure 3(b)) according to temperature and treatment time. The high effects of treatment time and temperature appear clearly and the coefficient values corresponding to these factors are very important. The results show that the increase of treatment time and temperature increases the real surface area and $\alpha$ as can be seen in Figure 3. To find the optimum via an alternative, the simplex sequential optimisation method was used. Experiment 8 was carried out at a point symmetrical to experiment 7 with respect to the midpoint between points 1 and 3 (Figure 4). Here, the response is high $\left(\mathrm{SS}=327 \mathrm{~m}^{2} \cdot \mathrm{m}^{-2}, \alpha=0.91\right)$, which shows that the increase of a treatment time and temperature allows the formation of conversion coating with high surface area.

3.3. Determination of Fractal Dimension of the Coating by Impedance Measurements. The electrochemical impedance diagrams of the conversion coating (Figure 5) show a capacitive arc characteristic of the charge transfer process at the electrode-solution interface. At very high frequencies, a process of diffusion in the pores is observed.

It has been shown that the transfer semicircle, which is centered for a flat smooth interface, becomes rotated around its high frequency when the surface is porous and/or rough. This difference from a smooth interface is due to the distribution of the system response time constant. The

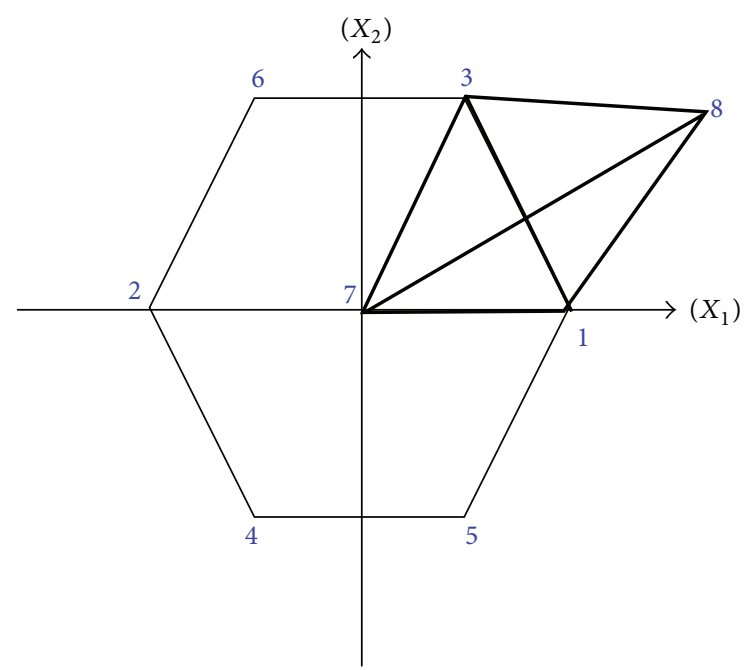

FIgURE 4: Illustration of simplex defined on $X_{1}$ (treatment time)$X_{2}$ (temperature).

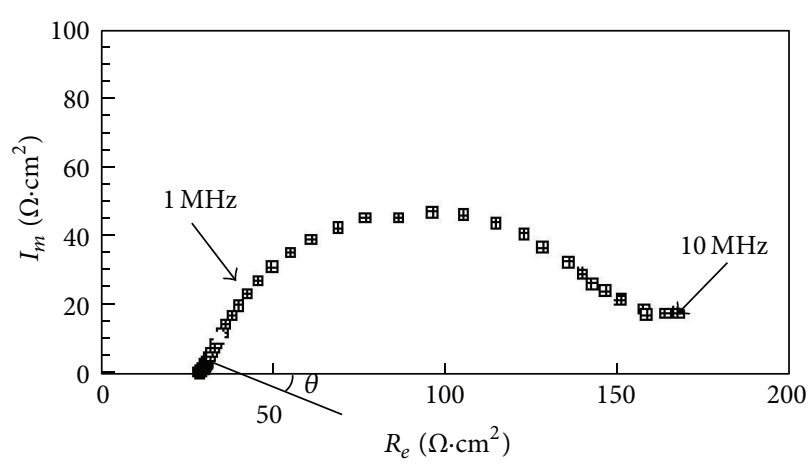

FIGURE 5: EIS Nyquist plot in aerated $1 \mathrm{M} \mathrm{Na}_{2} \mathrm{SO}_{4}$ aqueous solution of the austenitic stainless steel with conversion coating.

angle of rotation of capacitive loop around its high frequency is noted $\theta$ (Figure 5). In order to interpret any correlation that might exist between the particular texture of certain interfaces and the angle of rotation $\theta$, a nondimensional parameter $d_{f}$, representing the difference from an ideal surface (perfectly smooth and homogeneous), is often introduced. Several tentative relationships have been proposed to determine $d_{f}$ from the angle $\theta$ [16-21]. In this work, we used the relationship proposed by Le Mehauté and Crepy [16]:

$$
d_{f}=\frac{180}{180-2 \theta}+1
$$

For our optimal conversion coating, the obtained value of $d_{f}$ is about $2.15\left(\theta=12.2^{\circ}\right)$.

The double layer capacitance $C_{\mathrm{dl}}$ relevant to the EIS diagram in Figure 5 is $11775.3 \mu \mathrm{F} \cdot \mathrm{cm}^{-2}$, a high value attributed to the presence of a porous layer on the surface, while a double layer capacitance for a smooth surface is considered to be about $50 \mu \mathrm{F} \cdot \mathrm{cm}^{-2}$. The real surface area was estimated to be $235 \mathrm{~m}^{2} / \mathrm{m}^{2}$.

The calculated value of SS is in good agreement with what has been determined by cyclic voltammetry method. 


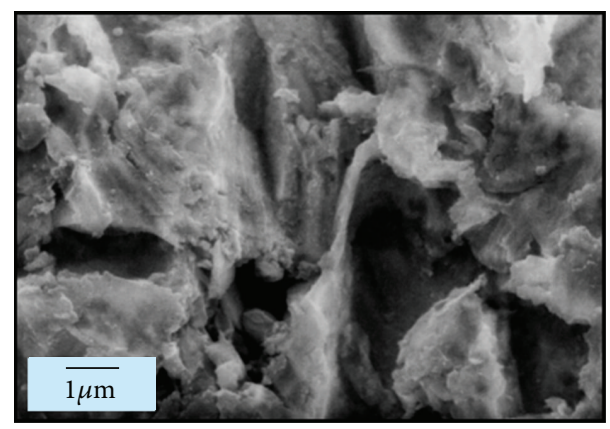

FIGURE 6: Micrograph of conversion coating obtained in an optimum bath.

Examination of the optimal coating by scanning electron microscopy showed that the surface is rough and porous (Figure 6).

\section{Conclusion}

The aim of this study is the optimisation of conversion coating on iron-chromium-nickel alloy. This conversion coating must have a high specific area. The present work has demonstrated that the experimental domain that we defined appears suitable for the optimisation of conversion coating. The fractional factorial design, Doehlert design, and simplex design allow a rapid overall study of conversion coating growth in sulphuric acid medium, under relatively strong experimental constraints. The primary conclusion of this study is that interactions between bath compounds and surface state have an important influence on the formation of conversion coatings. The experimental designs used for these experiments led to the optimum conditions being obtained. The real surface area of optimal conversion coating is very high $\left(235 \mathrm{~m}^{2} / \mathrm{m}^{2}\right)$. The fractal dimension was determined by impedance measurement. The measurements of specific area by impedance and cyclic voltammetry present a good agreement and indicate high porosity.

\section{References}

[1] P. Álvarez, A. Collazo, A. Covelo, X. R. Nóvoa, and C. Pérez, "The electrochemical behaviour of sol-gel hybrid coatings applied on AA2024-T3 alloy: effect of the metallic surface treatment," Progress in Organic Coatings, vol. 69, no. 2, pp. 175$183,2010$.

[2] Y. Song, D. Shan, R. Chen, F. Zhang, and E. Han, "A novel phosphate conversion film on Mg-8.8Li alloy," Surface and Coatings Technology, vol. 203, no. 9, pp. 1107-1113, 2009.

[3] A. A. Zuleta, E. Correa, C. Villada, M. Sepúlveda, J. G. Castaño, and F. Echeverría, "Comparative study of different environmentally friendly (Chromium-free) methods for surface modification of pure magnesium," Surface and Coatings Technology, vol. 205, no. 23-24, pp. 5254-5259, 2011.

[4] L. Bamoulid, M. T. Maurette, D. De Caro et al., "Investigations on composition and morphology of electrochemical conversion layer/titanium dioxide deposit on stainless steel," Surface and Coatings Technology, vol. 201, no. 6, pp. 2791-2795, 2006.
[5] L. Bamoulid, M.-T. Maurette, D. De Caro et al., "An efficient protection of stainless steel against corrosion: combination of a conversion layer and titanium dioxide deposit," Surface and Coatings Technology, vol. 202, no. 20, pp. 5020-5026, 2008.

[6] S. El Hajjaji, M. El Alaoui, P. Simon et al., "Preparation and characterization of electrolytic alumina deposit on austenitic stainless steel," Science and Technology of Advanced Materials, vol. 6, no. 5, pp. 519-524, 2005.

[7] A. Lgamri, A. Guenbour, A. Ben Bachir, S. El Hajjaji, and L. Aries, "Characterisation of electrolytically deposited alumina and yttrium modified alumina coatings on steel," Surface and Coatings Technology, vol. 162, no. 2-3, pp. 154-160, 2003.

[8] A. Komla, L. Aries, B. Naboulsi, and J. P. Traverse, "Texture of selective surfaces for photothermal conversion," Solar Energy Materials, vol. 22, no. 4, pp. 281-292, 1991.

[9] S. El Hajjaji, A. Lgamri, E. Puech-Costes, A. Guenbour, A. Ben Bachir, and L. Aries, "Optimization of conversion coatings: study of the influence of parameters with experimental designs," Applied Surface Science, vol. 165, no. 2, pp. 184-192, 2000.

[10] S. El Hajjaji, A. Guenbour, A. Ben Bachir, and L. Aries, "Effect of treatment baths nature on the characteristics of conversion coatings modified by electrolytic alumina deposits," Corrosion Science, vol. 42, no. 6, pp. 941-956, 2000.

[11] G. E. P. Box, W. G. Hunter, and J. S. Hunter, Statistics for Experimenters: An Introduction to Design, Data Analysis and Model Building, Wiley, New York, NY, USA, 1978.

[12] D. Mathieu and R. Phan-Tan-Luu, NEMROD Software, LPRAI, Marseille, France, 1995.

[13] D. H. Doehler, "Uniform shell designs," Journal of the Royal Statistical Society C, vol. 19, pp. 231-239, 1970.

[14] D. H. Doehlert and V. L. Klee, "Experimental designs through level reduction of the d-dimensional cuboctahedron," Discrete Mathematics, vol. 2, no. 4, pp. 309-334, 1972.

[15] W. Splendley, G. R. Hext, and F. R. Himsworth, "Sequential application of simplex design of optimization and evolutionary operations," Technometrics, vol. 4, pp. 441-461, 1962.

[16] A. Le Mehauté and G. Crepy, "Introduction to transfer and motion in fractal media: the geometry of kinetics," Solid State Ionics, vol. 9-10, pp. 17-30, 1983.

[17] M. Keddam and H. Takenouti, "Impedance of fractal interfaces : new data on the Von Koch model," Electrochimica Acta, vol. 33, pp. 445-448, 1986.

[18] A. J. Bard and L. R. Faulkner, Electrochimie, Masson, Paris, France, 1983.

[19] L. Nyikos and T. Pajkossy, "Fractal dimension and fractional power frequency-dependent impedance of blocking electrodes," Electrochimica Acta, vol. 30, pp. 1533-1540, 1985.

[20] T. Pajkossy and L. Nyikos, "Impedance of fractal blocking electrodes," Journal of Electrochemical Society, vol. 133, no. 10, pp. 2061-2064, 1986.

[21] S. H. Liu, "Fractal model for the ac response of a rough interface," Physical Review Letters, vol. 55, no. 5, pp. 529-532, 1985. 

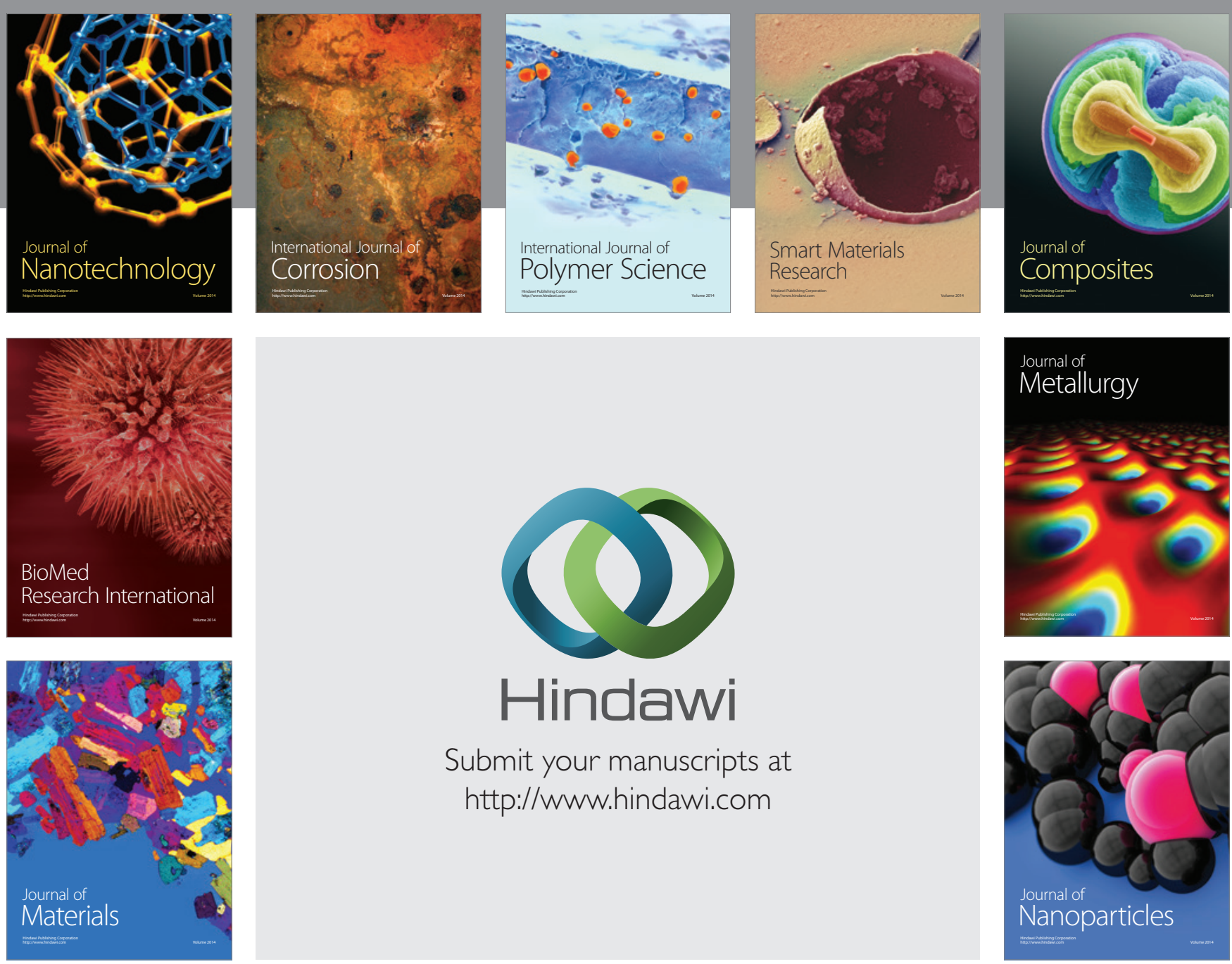

Submit your manuscripts at http://www.hindawi.com
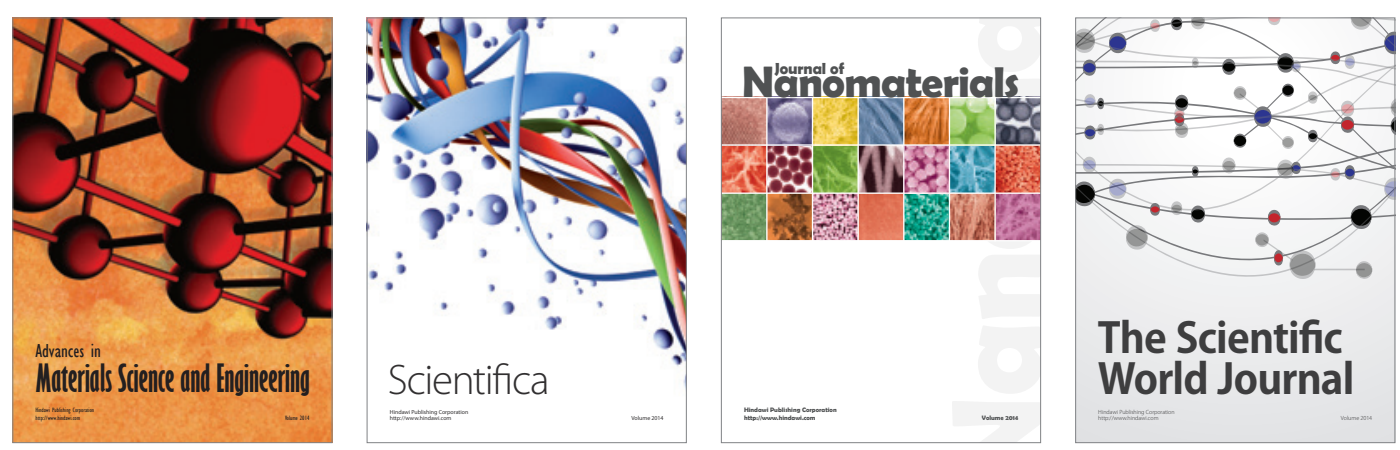

\section{The Scientific World Journal}
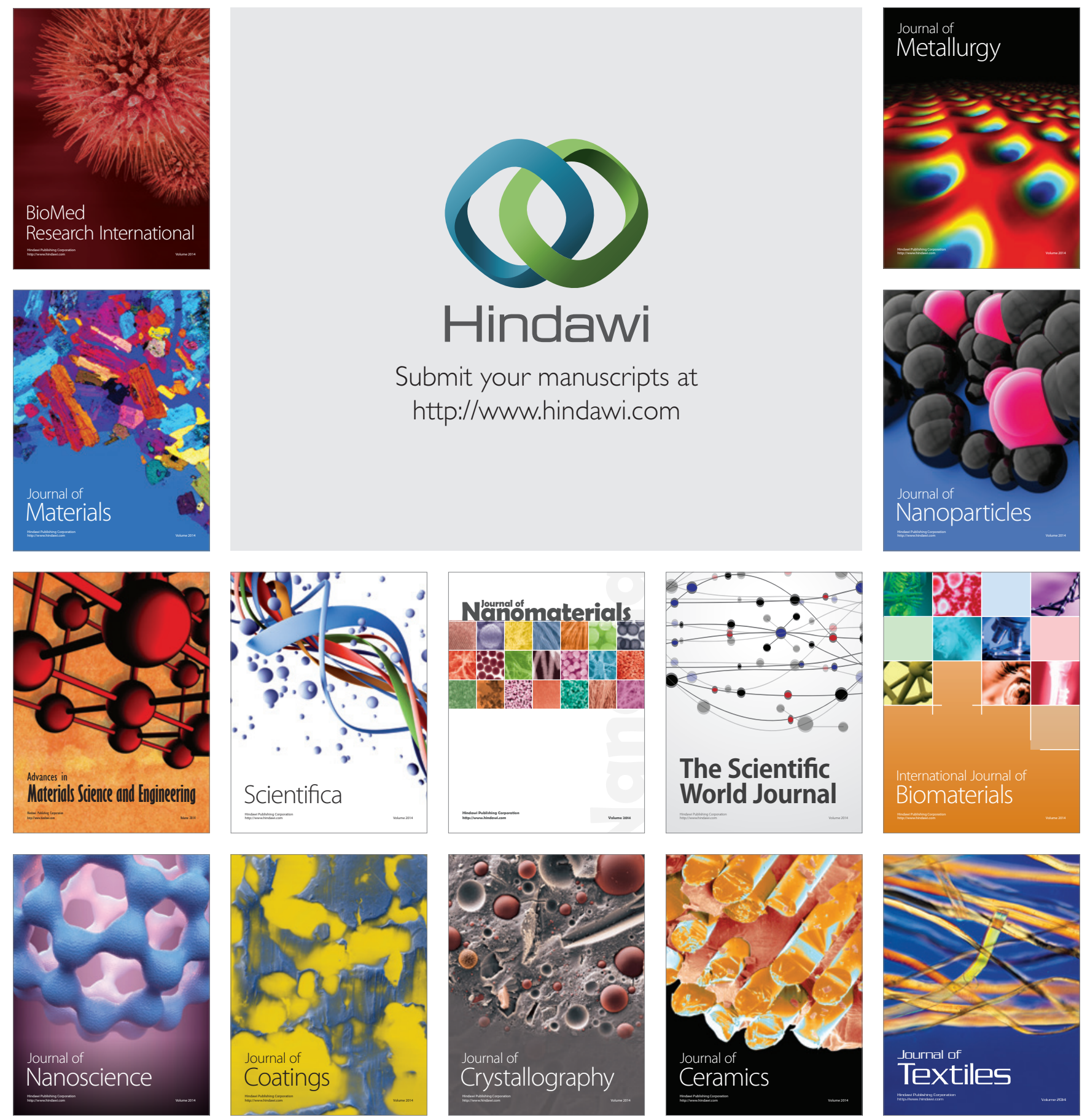